\title{
Bunnenberg, Christian/Steffen, Nils (Hrsg.): Geschichte auf YouTube. Neue Herausforderungen für Geschichtsvermittlung und Historische Bildung, 347 S., De Gruyter Oldenbourg, Berlin u.a. 2019.
}

\author{
Christine Gundermann
}

Angenommen: 29. April 2021 / Online publiziert: 22. Mai 2021

(C) Der/die Autor(en) 2021

Christian Bunnenberg und Nils Steffen präsentieren mit dem Band „Geschichte auf YouTube“ ein aktuelles Panorama der Erforschung, aber auch der Arbeit mit und der Erstellung von geschichtlichen Inhalten auf dem Videoportal YouTube. Die 14 von den Herausgebern versammelten Beiträge widmen sich dabei insbesondere dem Phänomen der Erklärvideos mit geschichtlichen Inhalten und werden von einer Einleitung und einem Ausblick der Herausgeber flankiert.

Die erste Sektion versammelt unter dem Zuschnitt „Kontexte“ drei Beiträge: Cord Arendes skizziert Entwicklungslinien vom Kinder- und Bildungsfernsehen der 1970er Jahre bis zu aktuellen Erklärvideos und definiert einführend den zentralen Untersuchungsgegenstand des Buches. Hier wäre es sicherlich weiterführend, moderne und hybride Erklärformate (etwa die „Chexperimente“ des KiKa), die ebenso im Fernsehen wie auf YouTube präsent sind, miteinzubeziehen, um medienspezifische Merkmale einerseits und Neuerungen im Bildungsbereich andererseits zu fokussieren. Nils Steffen argumentiert in seinem Beitrag, Erklärvideos vor allem als performative Historiografie wahrzunehmen, bei der die YouTuber_innen nicht nur die Rolle von Historiker_innen annehmen, sondern sie gleichzeitig über die Art und Weise ihrer Inszenierung ,performen“. Er arbeitet so heraus, dass die auf YouTube zugänglichen Erklärvideos nicht länger als Geschichtsvermittlung, sondern eher als Geschichtsdarstellung und -aneignung verstanden werden müssen (S. 63). Judith Uebing stellt in ihrem Beitrag eine überzeugende und belastbare Methodik zur Analyse von Erklärvideos mit geschichtlichen Inhalten vor, die sie anhand des Kanals „musstewissen Geschichte“ exemplarisch demonstriert und für interessierte Forschende als kurzgefasstes Fragenraster im Anhang des Beitrags bereitstellt.

C. Gundermann $(\bowtie)$

Universität Köln, Köln, Deutschland

E-Mail: christine.gundermann@uni-koeln.de 
Die zweite Sektion „Narrativität und Authentizität“ wird von Hannes Burkhardts Beitrag zur DDR-Geschichte auf YouTube eingeleitet. Auch er präsentiert exemplarisch eine Analysemethode, die es erlaubt, das Verhältnis von Zeitzeug_innen und Repräsentant_innen der Geschichtswissenschaft einerseits zu untersuchen und andererseits die präsentierte Geschichte auf ihre Funktion in der Gegenwart zu fokussieren. Christian Bunnenberg stellt in seinem Beitrag die Methode des Digital Storytelling anhand des partizipativen Projektes ,Stuttgart neu erzählt - Pulsierende Stadtgeschichten“ vor, welches er selbst mit umgesetzt hat. Benjamin Roers hingegen widmet sich dem Phänomen „MrWissen2go“ und untersucht die Authentifizierungsstrategien, die in den Angeboten des Kanals zu finden sind. Die überzeugende These, dass sich im vorliegenden Fall der „Presenter“, der die Geschichtswissenschaft präsentiert, als Zeitzeuge inszeniert oder zumindest als solcher gelesen werden kann, erscheint der Rezensentin besonders fruchtbar für weitere Forschungen zur Inszenierung von Geschichte auf YouTube. Weiterführend ist es hier sicherlich hilfreich, die im Text formulierte Objektauthentizität noch deutlicher mit Methoden wissenschaftlichen Arbeitens anzureichern - oder sich zugunsten der Betonung inszenatorischer Autorisierung von dieser zu lösen.

Die dritte Sektion „Produktion und Praxis“ lässt nun zwei Praktiker zu Wort kommen: Mirko Drotschmann aka „MrWissen2go“ (in Form eines Interviews) und Florian Wittig als „Creator“ des YouTube-Kanals „The Great War“. Beides sind wichtige Beiträge zum Verständnis der Produktionsbedingungen und des Selbstverständnisses der Akteur_innen im Netz. Sie helfen, Medienlogiken, wie etwa die von Wittig skizzierte Produktionsregel „Pics or it didn’t happen“ (S. 182), herauszuarbeiten. In beiden Beiträgen zeigt sich zudem deutlich das Spannungsfeld von Markenrepräsentation und Reflexion, indem aktive Praktiker_innen agieren, wenn sie sich am wissenschaftlichen Diskurs beteiligen.

In der Sektion „Partizipation“ präsentiert Henrike Rehders Überlegungen zu Potenzialen der Partizipation bei der Nutzung von YouTube. Ihre breite Analysen von Partizipationsformen im Netz bieten für alle weiteren Beiträge eine hilfreiche Grundlage, ihre Interpretationen von Geschichtskultur auf YouTube als ,neuer“ Zugang zu Geschichte und Erinnerung können aber nur bedingt überzeugen, denn wie sie selbst analysiert, wird Geschichte auf YouTube häufig auf positivistische Ereignisgeschichte reduziert und die damit angebotenen Narrative erscheinen der Rezensentin nicht als „optimale Lernumgebung“ (S. 204), gerade weil die hier diagnostizierte Multiperspektivität und vor allem die Kontroversität dem Ziel der Notenoptimierung beim nächsten Test geopfert werden. Moritz Hoffmann hingegen widmet sich der Historischen Hassrede auf YouTube als spezifischer Kategorie von geschichtskulturellen Phänomenen wie Holocaustleugnung und generellem Geschichtsrevisionismus und zeigt eindringlich, dass Historiker_innen als Teil einer engagierten Zivilgesellschaft, aber auch YouTube selbst, nur unzureichend mit diesem Phänomen umgehen können. Christoph Friedberg schließt die Buchsektion mit einem Beitrag zur Analyse von YouTube-Beiträgen über den Fokus „Nutzerrollen“ ab. Er versteht die Plattform dabei als Medium des Social Sharing und fragt nach Produzierenden, Bewertenden und Rezipierenden am Beispiel eines weloadtv-Beitrages zu Adolf Hitler.

Die fünfte Sektion ,YouTube und historische Bildung“ präsentiert die Analysen von Anja Neubert und dem Autor_innenteam Bernhard Linke und Marie Föl- 
len. Neubert seziert und vergleicht die Angebote des Kanals „TheSimpleClub“ und „musstewissen Geschichte“ exemplarisch auf das inszenierte Geschichtsverständnis der Produzierenden und stellt eine explorative Studie zur Wahrnehmung von Schüler_innen dieser Angebote vor. Linke und Föllen reflektieren in ihrem Beitrag den Einsatz von Erklärvideos für Studierende der Geschichtswissenschaft. In der finalen Sektion „Diskussion und Ausblick“ formuliert Jens Crueger Überlegungen zum Web 2.0 als Kulturellem Gedächtnis und die Herausgeber schließen mit einem Ausblick ab.

Der Band zeigt viele neue und überzeugende Wege auf, sich Geschichte auf YouTube analytisch zu nähern - die in der Gesamtschau sichtbare methodische Vielfalt ist damit eine der großen Stärken des Buches. In den Teilstudien werden zudem die Potenziale, aber auch die Herausforderungen einer partizipativen Geschichtskultur im Web 2.0 erörtert. Die Frage, wie genau eigentlich Geschichte im Format des Erklärvideos ,,erklärt“ wird, und was diese mediale und soziale Transformation historischer Angebote bedeutet, muss weiter untersucht werden. In diesem Sinne verweisen die Beiträge auch auf Leerstellen. So wird etwa in den Beiträgen ausschließlich von (weißen) Männern produzierte und präsentierte Geschichte vorgestellt - was diese Art der Vergeschlechtlichung von Geschichte aber bedeutet, wird in den einzelnen Beiträgen nicht thematisiert und durch eine teilweise fehlende gendergerechte Sprache im Band noch verstärkt (wohl aber im Ausblick als Leerstelle markiert). Gerade im Hinblick auf den in vielen Beiträgen herausgestellten generellen Trend, positivistische Geschichte auf YouTube zu erzählen, sollte hier weitergedacht werden.

Funding Open Access funding enabled and organized by Projekt DEAL.

Open Access Dieser Artikel wird unter der Creative Commons Namensnennung 4.0 International Lizenz veröffentlicht, welche die Nutzung, Vervielfältigung, Bearbeitung, Verbreitung und Wiedergabe in jeglichem Medium und Format erlaubt, sofern Sie den/die ursprünglichen Autor(en) und die Quelle ordnungsgemäß nennen, einen Link zur Creative Commons Lizenz beifügen und angeben, ob Änderungen vorgenommen wurden.

Die in diesem Artikel enthaltenen Bilder und sonstiges Drittmaterial unterliegen ebenfalls der genannten Creative Commons Lizenz, sofern sich aus der Abbildungslegende nichts anderes ergibt. Sofern das betreffende Material nicht unter der genannten Creative Commons Lizenz steht und die betreffende Handlung nicht nach gesetzlichen Vorschriften erlaubt ist, ist für die oben aufgeführten Weiterverwendungen des Materials die Einwilligung des jeweiligen Rechteinhabers einzuholen.

Weitere Details zur Lizenz entnehmen Sie bitte der Lizenzinformation auf http://creativecommons.org/ licenses/by/4.0/deed.de. 